\title{
Corrigendum
}

\section{Team-Based Learning, a Learning Strategy for Clinical Reasoning, in Students with Problem-Based Learning Tutorial Experiences}

\author{
Yumiko Okubo, ${ }^{1}$ Naoko Ishiguro, ${ }^{2}$ Taiyo Suganuma, ${ }^{1}$ Toshio Nishikawa, ${ }^{3}$ \\ Toshio Takubo, ${ }^{4}$ Noriko Kojimahara, ${ }^{5}$ Rie Yago, ${ }^{6}$ Shinichi Nunoda, ${ }^{7}$ \\ Shigetaka Sugihara ${ }^{8}$ and Toshimasa Yoshioka ${ }^{1}$

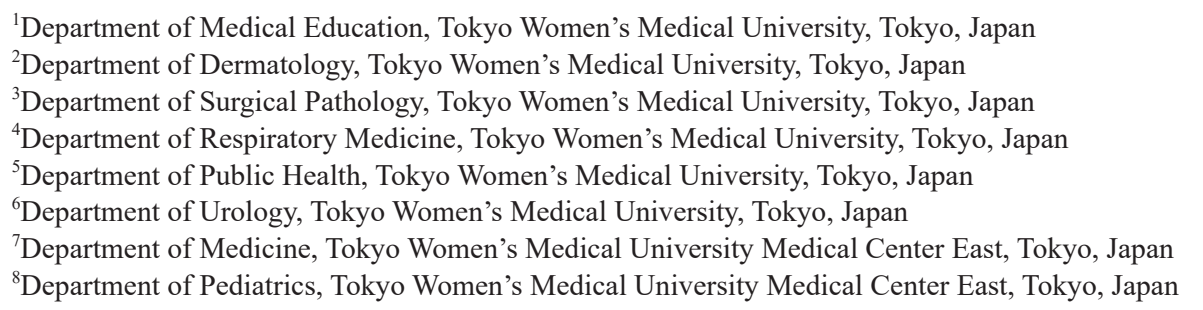

Tohoku J. Exp. Med., 227: 23-29, 2012.

In the "Results" section (page 25) of this article published in the May issue, 2012, the authors found the mistakes in the description of Fig. 3B: "total CBT scores (Fig. 3B), non-TBL, $\mathrm{n}=298,76.2 \pm 7.5$, TBL, $\mathrm{n}=308,44.5 \pm$
7.4) ( $<$ 0.05)." The corrected text is "total CBT scores (Fig. 3B, non-TBL, $\mathrm{n}=298,76.2 \pm 7.5$, TBL, $\mathrm{n}=308,77.2$ $\pm 8.6)(\mathrm{p}<0.05)$." We apologize for such mistakes in the description. 